\title{
Finite Gap Jacobi Matrices and the Schottky-Klein Prime Function
}

\author{
Darren Crowdy ${ }^{1}$
}

Received: 12 July 2015 / Revised: 10 May 2016 / Accepted: 16 September 2016 /

Published online: 8 November 2016

(C) The Author(s) 2016. This article is published with open access at Springerlink.com

\begin{abstract}
A covering map formalism for studying the spectral curves associated with finite gap Jacobi matrices is presented. We advocate a constructive function theoretic framework based on use of the Schottky-Klein prime function. The single gap, or genus-one, case is studied in explicit detail.
\end{abstract}

Keywords Jacobi matrix · Finite gap · Isospectral torus · Schottky-Klein prime function

Mathematics Subject Classification $30 \mathrm{C} 20 \cdot 31 \mathrm{~A} 15$

\section{Introduction}

This paper is motivated by recent work of Christiansen et al. [9] who describe a covering map formalism to define and study the isospectral torus associated with finite gap Jacobi matrices. In turn, that paper drew on earlier work of Sodin and Yuditskii [29] and Peherstorfer and Yuditskii [27] which concentrated on infinite gap sets. The focus in the present paper is the finite gap situation, and our goals are limited to offering a complementary perspective to the covering map formalism of prior work. It is offered both for mathematical interest and because it is believed that the approach here provides a framework for constructive computational methods.

Communicated by Alexander Aptekarev.

$凶$ Darren Crowdy

d.crowdy@imperial.ac.uk

1 Department of Mathematics, Imperial College London, 180 Queen's Gate, London SW7 2AZ, UK 
In another work, the author has been interested in physical science problems involving arbitrary multiply connected domains, that is, finitely connected planar domains generally not possessing any geometrical symmetries. The mathematical approach has been to develop a constructive framework based on the Schottky uniformization of the so-called Schottky double-a compact Riemann surface naturally associated to any given multiply connected planar domain - and to make use of the Schottky-Klein prime function that lives on that surface as the basic functional building block for more complicated functions (we adopt the designation "Schottky-Klein prime function" following Baker [3]; see also [7,8]). This idea has proven to be surprisingly fruitful. A survey of many other results, including applications to potential theory, the construction of multiply connected quadrature domains, the solution to a variety of problems in fluid dynamics, and the derivation of a multiply connected extension of the classical Schwarz-Christoffel conformal mapping formula, is given in the review articles [10,11]. Most recently, a connection between a problem in the field of vortex dynamics and the planar limit of random normal matrices has been pointed out [19]; both those application areas benefit from the function theoretic approach just outlined, either for the description of vortex patches ("V-states") or, in the random matrix case, the limiting shapes of the multi-support eigenvalue sets.

The central point of contact with finite gap Jacobi matrices is the observation that the hyperelliptic curve associated with a given multi-support spectrum can be viewed as the Schottky double of the multiply connected domain exterior to a finite set of bands on the real axis. For general multiply connected domains, not necessarily enjoying any reflectional symmetry about the real axis, the Schottky double is made up of two "sides", often referred to as a frontside and a backside, with an antiholomorphic involution relating them [24]. With an additional reflectional symmetry about the real axis, the natural holomorphic coordinate on the backside of the double becomes the same as that on the frontside and the Schottky double is a two-sheeted hyperelliptic Riemann surface which we call $\mathcal{S}$ following [9]. The two sheets of the surface will be denoted by $\mathcal{S}^{+}$(the "physical" sheet) and $\mathcal{S}^{-}$. Accordingly, the points at infinity on the two sheets will be denoted by $\infty^{+}$and $\infty^{-}$.

To fix ideas, a (one-sided) Jacobi matrix $J$ as considered in [9] is one of the tridiagonal forms

$$
J=\left[\begin{array}{rrrrrr}
b_{1} & a_{1} & 0 & 0 & 0 & \cdots \\
a_{1} & b_{2} & a_{2} & 0 & 0 & \cdots \\
0 & a_{2} & b_{3} & a_{3} & 0 & \cdots \\
0 & 0 & a_{3} & b_{4} & a_{4} & \cdots \\
\vdots & \vdots & \vdots & \vdots & \vdots &
\end{array}\right]
$$

The key mathematical results of this paper can be summarized concisely as follows. Let the essential spectrum of such a Jacobi matrix take the form

$$
\begin{aligned}
\mathbf{e} & =\mathbf{e}_{\mathbf{0}} \cup \mathbf{e}_{2} \cup \cdots \cup \mathbf{e}_{\mathbf{l}}, \\
\mathbf{e}_{\mathbf{j}} & =\left[\alpha_{j}, \beta_{j}\right], \\
\alpha_{0} & <\beta_{0}<\alpha_{1}<\cdots<\alpha_{l}<\beta_{l}
\end{aligned}
$$


Fig. 1 A typical finite gap spectrum on the real axis with $l=2$

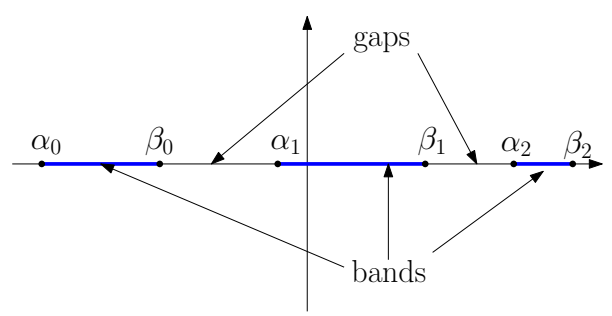

which is a union of $l+1$ disjoint closed intervals, or bands, with $l$ gaps. The case $l=2$ is shown schematically in Fig. 1. It is known that the isospectral torus of a Jacobi matrix having such a spectrum can be parametrized in terms of so-called minimal Herglotz functions [9]; the isospectral torus essentially encodes the set of Jacobi matrices having the same essential spectrum. We show here that a parametrization of a typical minimal Herglotz function $m(z)$ associated to such a finite gap set is furnished, in terms of a parameter $\zeta$, by the explicit formulas

$$
\begin{aligned}
& z=f(\zeta)=A \frac{\omega(\zeta, \beta) \omega(\zeta, 1 / \beta)}{\omega(\zeta, \alpha) \omega(\zeta, 1 / \alpha)} \\
& m=M(\zeta)=D\left[\frac{\omega(\zeta, \alpha)}{\omega(\zeta, 1 / \alpha)}\right] \prod_{j=1}^{l}\left[\frac{\omega\left(\zeta, s_{j}\right)}{\omega\left(\zeta, r_{j}\right)}\right] \mathrm{e}^{2 \pi i \sum_{k=1}^{l} n_{k} v_{k}(\zeta)}
\end{aligned}
$$

where $\omega(.,$.$) is the Schottky-Klein prime function associated to a multiply connected$ circular domain $D_{\zeta}$ consisting of the unit $\zeta$-disc with $l$ smaller circular discs excised, all with centres located on the real diameter $(-1,1) . A$ and $D$ are constants, the set $\left\{r_{j} \mid j=1, \ldots, l\right\}$ gives the $l$ poles of the minimal Herglotz function, while $\left\{s_{j} \mid j=\right.$ $1, \ldots, l\}$ is a set of its zeros. Another pole at the real point $\alpha$ corresponds to $\infty^{+}$. The exponential terms appearing in $M(\zeta)$ will be explained later in the paper.

Based on these results, we find the following explicit expressions that assist in the inverse problem of finding the Jacobi parameters associated to e, as explained later. Namely

$$
\begin{aligned}
& b_{1}=\left.X_{\infty} \frac{\mathrm{d}}{\mathrm{d} \zeta} \log N(\zeta)\right|_{\zeta=\alpha}, \\
& a_{1}^{2}=Y_{\infty} b_{1}-\frac{b_{1}^{2}}{2}+\left.\frac{X_{\infty}^{2}}{2} \frac{\mathrm{d}^{2}}{\mathrm{~d} \zeta^{2}} \log N(\zeta)\right|_{\zeta=\alpha}
\end{aligned}
$$

where

$$
N(\zeta) \equiv\left[\frac{\omega(\zeta, \beta) \omega(\zeta, 1 / \beta)}{\omega(\zeta, 1 / \alpha)^{2}}\right] \prod_{j=1}^{l}\left[\frac{\omega\left(\zeta, s_{j}\right)}{\omega\left(\zeta, r_{j}\right)}\right] \mathrm{e}^{2 \pi i \sum_{k=1}^{l} n_{k} v_{k}(\zeta)}
$$

is meromorphic on $\mathcal{S}$ (it is automorphic under a Schottky group action associated to $\left.D_{\zeta}\right)$. The set of zeros $\left\{s_{j} \mid j=1, \ldots, l\right\}$ is given by the solution of a system of equations written down in Sect. 6 . The parameter $X_{\infty}$ is related to the logarithmic capacity of 
e while $Y_{\infty}$ is a geometrical parameter (explained later) associated with the spectral bands. Formulas for both are also available in terms of the $\mathrm{S}-\mathrm{K}$ prime function and a related function $\tilde{\omega}(\zeta, \alpha)$ defined later in (28):

$$
X_{\infty}=A \frac{\omega(\alpha, \beta) \omega(\alpha, 1 / \beta)}{\tilde{\omega}(\alpha, \alpha) \omega(\alpha, 1 / \alpha)} \quad Y_{\infty}=\left.A\left\{\frac{\mathrm{d}}{\mathrm{d} \zeta}\left[\frac{\omega(\zeta, \beta) \omega(\zeta, 1 / \beta)}{\tilde{\omega}(\zeta, \alpha) \omega(\zeta, 1 / \alpha)}\right]\right\}\right|_{\zeta=\alpha}
$$

The Schottky double setting has been employed previously by Aptekarev [2] in a study of orthogonal polynomials on a general set of contours, but he builds the associated function theory based on a Riemann theta function formalism. Here, we eschew the latter and use instead the Schottky-Klein prime function as the basic building block in the belief that it is a natural way to build the requisite functions. Lukashov and Peherstorfer [22] (see also [7,8]) have used a function theory similar to ours in their studies of orthogonal polynomials (although they use the designation Schottky-Burnside function to refer to what we call the Schottky-Klein prime function). Christiansen, Simon and Zinchenko [9] also avoid use of the Riemann theta function and develop their own construction based on representations of the requisite functions in terms of infinite Blaschke products. Additional discussion of the Schottky model and its applications in approximation theory is given in the monograph by Bogatyrëv [6].

It is worth clarifying how our approach differs from previous work since it allows us to underline the reason for writing this article. Like [9], we adopt a covering map formalism but our viewpoint differs in that it is the bands, not the gaps, that correspond to Möbius-identified circles in our uniformization domain. But the most important distinction is that, within this alternative model of the underlying curves, the function theory we employ to solve the inverse problem is different to that used in previous work: here we use the Schottky-Klein prime function which affords many constructive advantages. This is because the efficient computation of $\mathrm{S}-\mathrm{K}$ prime functions has been a focus of much recent work by the author and co-workers who have prepared freely downloadable software, on the MATLAB platform, for its fast and effective computation $[15,20]$. Further discussion of this is given in Sect. 11.

\section{The Schottky Double of a Domain}

It is known, by a multiply connected extension of the Riemann mapping theorem [23], that any $(M+1)$-connected domain (for $M \geq 0$ ) is conformally equivalent to the unit $\zeta$-disc with $M$ smaller circular discs excised. Let such a domain be denoted $D_{\zeta}$. To model the so-called Schottky double of $D_{\zeta}$ one can reflect, in the unit circle, the circular boundaries of the interior discs to produce $M$ additional circles in $|\zeta|>1$. By this same antiholomorphic reflection of points in $D_{\zeta}$, one produces a precise copy, or "backside", of the original multiply connected domain outside the unit $\zeta$-disc. We then identify each circle inside $|\zeta|<1$ with its reflection in $|\zeta|>1$ by means of a holomorphic Möbius transformation. For an $(M+1)$-connected domain there will be $M$ such Möbius transformations. The basic idea of this construction lies at the heart of the Schottky model of algebraic curves and, by forming the union of $D_{\zeta}$ 
with its backside (its reflection in the unit circle) and identifying the boundary circles as just described, we produce a model of the Schottky double. Gustafsson [24] and Varchenko and Etingof [30] have discussed the so-called Hele-Shaw free boundary problem in a multiply connected domain by considering the Schottky double of the domain. Aptekarev [2] used it in his study of orthogonal polynomials on a general set of contours.

In more detail, suppose the $M$ smaller circular discs inside $|\zeta|<1$ have centres $\left\{\delta_{j} \mid j=1, \ldots, M\right\}$ and radii $\left\{q_{j} \mid j=1, \ldots, M\right\}$. The data $\left\{\delta_{j}, q_{j} \mid j=1, \ldots, M\right\}$ will be called the conformal moduli of $D_{\zeta}$ and they can be uniquely associated to a given set of spectral bands up to specification of an automorphism of the unit disc. Let the unit circle be denoted $C_{0}$ and let the $M$ interior circular boundaries be denoted by $\left\{C_{k} \mid k=1, \ldots, M\right\}$. For $k=1, \ldots, M$, let $C_{k}^{\prime}$ denote the reflection of $C_{k}$ in $C_{0}$. Figure 2 shows a schematic in the quadruply connected case $M=3$. For $k=0,1, \ldots, M$, introduce the Möbius transformation $\phi_{k}(\zeta)$ defined by

$$
\phi_{k}(\zeta)=\overline{\delta_{k}}+\frac{q_{k}^{2}}{\zeta-\delta_{k}}, \quad k=0,1, \ldots, M
$$

It is straightforward to check that for points on the circle $C_{k}$

$$
\phi_{k}(\zeta)=\bar{\zeta}
$$

We define the reflection of a point $\zeta$ in the circle $C_{k}$ by $\overline{\phi_{k}(\zeta)}$. Then for $k=1, \ldots, M$ introduce the Möbius transformation $\theta_{k}(\zeta)$ defined by

$$
\theta_{k}(\zeta)=\overline{\phi_{k}\left(\bar{\zeta}^{-1}\right)}, \quad k=1, \ldots, M
$$

It follows from (9) and (7) that

$$
\theta_{k}(\zeta)=\delta_{k}+\frac{q_{k}^{2} \zeta}{1-\overline{\delta_{k}} \zeta}, \quad k=1, \ldots, M
$$

It is straightforward to show that $\theta_{k}(\zeta)$ maps $C_{k}^{\prime}$ holomorphically onto $C_{k}$ as illustrated schematically in Fig. 2.

The set $\Theta$ consisting of all functional compositions of the maps $\left\{\theta_{k}(\zeta) \mid k=\right.$ $1, \ldots, M\}$ and their inverses, including the identity map, is an example of a classical Schottky group $[3,4]$. We refer to the maps $\left\{\theta_{k}(\zeta) \mid k=1, \ldots, M\right\}$ and their inverses as the generators of the group $\Theta$. A fundamental region of $\Theta$ is a connected region whose images under all maps in $\Theta$ tessellate the complement of the limit set of the group. Let us define $F$ as the region consisting of $D_{\zeta}$ and its reflection in $C_{0}$, i.e. the $2 M$-connected region bounded by $\left\{C_{k}, C_{k}^{\prime} \mid k=1, \ldots, M\right\}$. Then $F$ is a fundamental region for the group $\Theta$. 


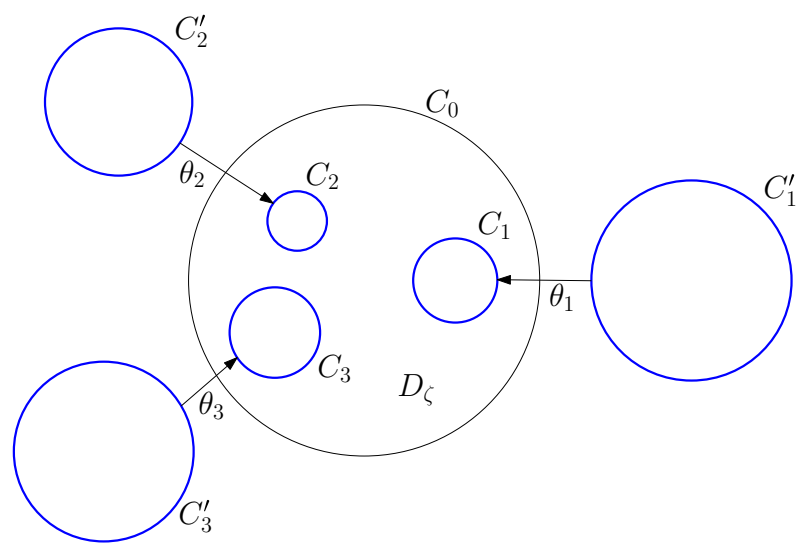

Fig. 2 Model of a general Schottky double with $M=3$. The circles $\left\{C_{j}, C_{j}^{\prime} \mid j=1, \ldots, M\right\}$ and the maps $\left\{\theta_{j}(\zeta) \mid j=1, \ldots, M\right\}$ identifying them. $C_{0}$ is the unit circle that separates the two sides of the double. For Jacobi matrices with a real spectrum all circles are centred on the real axis

\section{The Schottky-Klein Prime Function}

Associated with the Schottky double of the given domain $D_{\zeta}$ are $M$ integrals of the first kind [3] which we denote $\left\{v_{k}(\zeta) \mid k=1, \ldots, M\right\}$. These are analytic, but not single valued, in $F$. Indeed, for $j, k=1, \ldots, M$, we fix the normalization [3]

$$
\left[v_{k}(\zeta)\right]_{C_{j}}=-\left[v_{k}(\zeta)\right]_{C_{j}^{\prime}}=\delta_{j k}
$$

where $\left[v_{k}(\zeta)\right]_{C_{j}}$ and $\left[v_{k}(\zeta)\right]_{C_{j}^{\prime}}$ denote, respectively, the changes in $v_{k}(\zeta)$ on traversing $C_{j}$ and $C_{j}^{\prime}$ with the interior of $F$ on the right. $\delta_{j k}$ denotes the Kronecker delta function. Then, for $j, k=1, \ldots, M$ and modulo the addition of integers dependent on the choice of integration paths for $\left\{\mathrm{d} v_{k} \mid k=1, \ldots M\right\}$, we have

$$
v_{k}\left(\theta_{j}(\zeta)\right)-v_{k}(\zeta)=\tau_{j k}
$$

for some $\left\{\tau_{j k} \mid j, k=1, \ldots, M\right\}$ which are constants (i.e. they are independent of $\zeta$, but they depend on the conformal moduli).

It is established in [25] that there exists a unique function $X(\zeta, \alpha)$ defined by the properties:

(i) $X(\zeta, \alpha)$ is single valued and analytic in $F$ (except possibly at infinity if this point is contained in $F$ ).

(ii) $X(\zeta, \alpha)$ has a second-order zero at each of the points $\theta(\alpha), \theta \in \Theta$.

(iii) It is normalized such that

$$
\lim _{\zeta \rightarrow \alpha} \frac{X(\zeta, \alpha)}{(\zeta-\alpha)^{2}}=1
$$


(iv) If $\theta_{k}(\zeta)$ (for $k=1, \ldots, M$ ) is one of the generators of $\Theta$, then

$$
X\left(\theta_{k}(\zeta), \alpha\right)=\exp \left(-2 \pi \mathrm{i}\left(2 v_{k}(\zeta)-2 v_{k}(\alpha)+\tau_{k k}\right)\right) \frac{\mathrm{d} \theta_{k}(\zeta)}{\mathrm{d} \zeta} X(\zeta, \alpha)
$$

The Schottky-Klein prime function (henceforth, S-K prime function) is then defined as the square root of $X(\zeta, \alpha)$. There is a similar characterization for the prime function itself subject only to an ambiguity in sign, a matter which has recently been discussed by Bogatyrëv [5].

For the special class of Schottky groups considered here, it is also possible to establish [14,31] that the corresponding S-K prime function and its Schwarz conjugate function satisfy the additional functional relation

$$
\bar{\omega}(1 / \zeta, 1 / \alpha)=-\frac{1}{\zeta \alpha} \omega(\zeta, \alpha)
$$

where the Schwarz conjugate $\bar{h}(\zeta)$ of an analytic function $h(\zeta)$ is defined by $\bar{h}(\zeta) \equiv$ $\overline{h(\bar{\zeta})}$.

A classical infinite product formula of the Schottky-Klein prime function is $[3,13]$

$$
\omega(\zeta, \alpha)=(\zeta-\alpha) \prod_{\theta_{i} \in \Theta^{\prime \prime}} \frac{\left(\theta_{i}(\zeta)-\alpha\right)\left(\theta_{i}(\alpha)-\zeta\right)}{\left(\theta_{i}(\zeta)-\zeta\right)\left(\theta_{i}(\alpha)-\alpha\right)}
$$

where $\theta_{i}(\zeta)$ is taken to be all elements in the set, denoted by $\Theta^{\prime \prime}$, comprising all elements of the group $\Theta$ excluding the identity and all inverses. While this formula has been used in applications $[13,14]$ to compute the prime function it can be inefficient to implement numerically especially as the connectivity of the domains grows. It is for this reason that the author, with collaborators, has developed novel numerical algorithms for the evaluation of the prime function that do not rely on this classical infinite product formula [15,20]. Indeed an important fact, with both theoretical and numerical implications (cf. Crowdy and Marshall [15]), is that all the symmetries of Schottky doubles of planar domains imply that the relevant integrals of the firstkind $\left\{v_{k}(\zeta) \mid k=1, \ldots, M\right\}$ are linear combinations of the analytic extensions of the harmonic measures of the domain (defined below). Crowdy and Marshall [15] used this fact to devise a novel and effective numerical algorithm to compute both the $\left\{v_{k}(\zeta) \mid k=1, \ldots, M\right\}$ - and hence, in effect, the Abel map-as well as the S-K prime function itself, and without recourse to the (often slowly convergent) infinite product formula (16). The algorithms of [15] have now been improved even further [20].

In more detail, it is known [15] that $\operatorname{Im}\left[v_{j}(\zeta)\right]=0$ on $|\zeta|=1$ so that by analytic continuation off this circle

$$
\overline{v_{j}}(1 / \zeta)=v_{j}(\zeta)
$$

Now introduce the modified functions

$$
\tilde{v}_{j}(\zeta)=H_{j}(\zeta)+\mathrm{i} \Omega_{j}(\zeta)
$$


where $\left\{\Omega_{j} \mid j=1, \ldots, l\right\}$ are what we refer to as the harmonic measures [15] of the domain: for any $j=1, \ldots, M$, the function $\Omega_{j}$ is harmonic everywhere in the fundamental region and satisfies the boundary conditions

$$
\Omega_{j}= \begin{cases}1, & \text { on } C_{j}, \\ 0, & \text { on } C_{k}, \quad k \neq j .\end{cases}
$$

$H_{j}(\zeta)$ is the harmonic conjugate of $\Omega_{j}$. It is demonstrated in [15] that we can write

$$
v_{j}(\zeta)=\sum Q_{j k} \tilde{v}_{k}(\zeta)
$$

for some symmetric invertible matrix $Q_{j k}$ where, in fact [15],

$$
Q_{j k}=\frac{i \tau_{j k}}{2}
$$

where $\tau_{j k}$ appeared earlier in (12).

\section{Potential Theory}

In their development Christiansen, Simon and Zinchenko [9] make connections between the inverse Jacobi problem and the potential theory associated with the spectral bands. To make contact with those observations, we introduce a few additional ideas (Fig. 3).

For an $M$-connected domain $D_{\zeta}$ there are $M+1$ so-called modified Green's functions $\left\{G_{j}(\zeta, \alpha) \mid j=0,1, \ldots M\right\}$. The function $G_{j}(\zeta, \alpha)$ has a logarithmic singularity in $D_{\zeta}$ at $\alpha$, vanishes on boundary $C_{j}$, and has a constant value on all other boundaries of $D_{\zeta}$ [28]; those constants are determined by the conditions that

$$
\oint_{C_{k}} \frac{\partial G_{j}}{\partial n} \mathrm{~d} s=0, \quad k=1, \ldots, M, \quad k \neq j,
$$

Fig. 3 Preimage circular domain $D_{\zeta}$ in the $\zeta$ plane: the unit circle with two excised circles with centres $\delta_{1}$ and $\delta_{2}$ on the real axis. Their radii are $q_{1}$ and $q_{2}$. The real point $\alpha$ maps to $\infty^{+}$; its reflection $1 / \alpha$, which sits outside the unit disc, corresponds to $\infty^{-}$

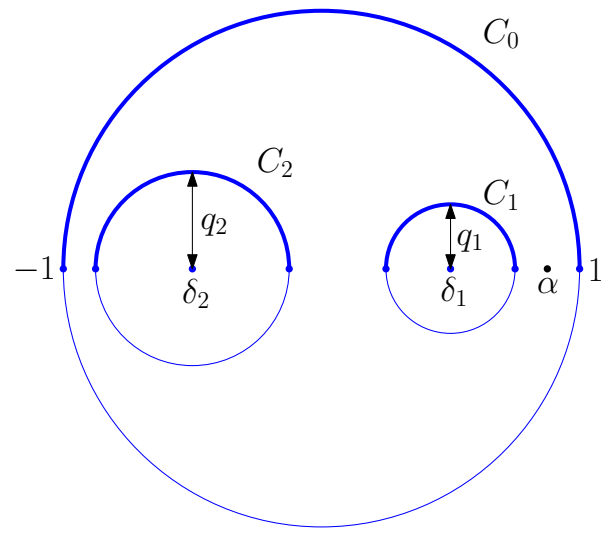


where $s$ denotes boundary arc length and $\partial / \partial n$ is the normal derivative. These modified Green's functions are distinct from the usual potential theoretic first-type Green's function which has zero imaginary part on all boundaries (and does not, in general, satisfy (22)).

Within our Schottky model of the hyperelliptic curve, the analytic extension $\tilde{G}_{0}(\zeta, \alpha)$ of a modified Green's function $G_{0}(\zeta, \alpha)$ for our domain has relevance to the inverse Jacobi problem. It was demonstrated in $[14,18]$ that the analytic extension $\tilde{G}_{0}(\zeta, \alpha)$ of a multiply connected domain $D_{\zeta}$ has, in terms of the associated S-K prime function, the concise representation

$$
\tilde{G}_{0}(\zeta, \alpha)=\frac{1}{2 \pi \mathrm{i}} \log \left[\frac{\omega(\zeta, \alpha)}{|\alpha| \omega(\zeta, 1 / \bar{\alpha})}\right] .
$$

It follows from (23) that

$$
\frac{\omega(\zeta, \alpha)}{|\alpha| \omega(\zeta, 1 / \bar{\alpha})}=\mathrm{e}^{2 \pi \mathrm{i} \tilde{G}_{0}(\zeta, \alpha)}
$$

and it is this fact that will be useful later.

\section{Conformal Mapping to $l+1$ Real Slits}

All of the mathematical technologies we have introduced so far pertain to any multiply connected domain of connectivity $M+1$. Henceforth, we set $M=l$, in accord with the notation of [9] where the spectrum of a Jacobi matrix is taken to comprise $l+1$ bands on the real axis with $l$ finite gaps. Only now do we restrict to the special class of domains $D_{\zeta}$ for which all the circles $\left\{C_{j} \mid j=1, \ldots, l\right\}$ are centred on the real interval $(-1,1)$.

We wish to construct a conformal map from $D_{\zeta}$ to the unbounded region exterior to the $l+1$ bands e and where the circular boundary $C_{j}$ is transplanted to the band $\left[\alpha_{j}, \beta_{j}\right]$ for $j=0,1, \ldots, l$. Portions of the real $\zeta$-axis inside the unit disc will be transplanted to the gaps with some point $\alpha$ chosen to map to $z=\infty$. By the freedoms of the Riemann mapping theorem, the point $\alpha$ can be chosen as we like provided it lies on the real $\zeta$-axis outside the circles $\left\{C_{k} \mid k=1, \ldots, l\right\}$ and inside $C_{0}$.

The required conformal mapping is provided by an instance of the so-called "radial slit mapping" formula

$$
z=f(\zeta)=A \frac{\omega(\zeta, \beta) \omega(\zeta, 1 / \bar{\beta})}{\omega(\zeta, \alpha) \omega(\zeta, 1 / \bar{\alpha})}
$$

written down in terms of the S-K prime function by Crowdy and Marshall [12]; see also [22] where a similar result is recorded in a different context. For the present application, we require $\alpha, \beta \in \mathbb{R}$. It is easy to show from the symmetry property (15) of the S-K prime function that (25) in fact maps the other half of $F$ (i.e. the reflection of $D_{\zeta}$ in the unit circle) onto another copy of the plane exterior to the slits.

The explicit form (25) means that once a domain $D_{\zeta}$ is fixed, so that the conformal modulus set $\left\{q_{j}, \delta_{j} \mid j=1, \ldots, l\right\}$ and the parameters $A, \alpha$ and $\beta$ are known, formulas 
for the finite gap set $\mathbf{e}$ follow directly from the formulas

$$
\alpha_{j}=f\left(\delta_{j}+q_{j}\right), \quad \beta_{j}=f\left(\delta_{j}-q_{j}\right), \quad j=0,1, \ldots, l .
$$

Conversely, if the finite gap set $\mathbf{e}$ is given then it is a simple numerical matter to inverse the explicit non-linear relations (26) to find the relevant conformal moduli.

Furthermore, from a Laurent expansion of (25) near $\zeta=\alpha$ we have that, locally,

$$
z \sim \frac{X_{\infty}}{(\zeta-\alpha)}+Y_{\infty}+\cdots
$$

for some constants $X_{\infty}$ and $Y_{\infty}$. Indeed, if we define the function $\tilde{\omega}(\zeta, \alpha)$ via

$$
\omega(\zeta, \alpha)=(\zeta-\alpha) \tilde{\omega}(\zeta, \alpha)
$$

then we find the formulas (6). The parameter $X_{\infty}$ is related to the conformal radius or logarithmic capacity of the domain. Immediately we observe that, near $z=\infty$ or $\zeta=\alpha$,

$$
(\zeta-\alpha)=\frac{X_{\infty}}{z}+\frac{X_{\infty} Y_{\infty}}{z^{2}}+\cdots, \quad(\zeta-\alpha)^{2}=\frac{X_{\infty}^{2}}{z^{2}}+\cdots
$$

The function (25) is invariant under the action of the Schottky group $\Theta$, i.e. it is automorphic with respect to this group action. This can be argued directly from its required properties as the relevant conformal mapping function where the image of each circle $C_{k}$ (for $k=0,1, \ldots, l$ ) is required to lie on the real $z$-axis. Alternatively, given the explicit form (25), it can also be verified directly on use of the properties (14) of the S-K prime function.

Although we do not make use of them here, we record that there are at least two alternative ways to write the conformal map taking $D_{\zeta}$ to $\mathcal{S}_{+}$in terms of the associated $\mathrm{S}-\mathrm{K}$ prime function. We could also write it as the "parallel slit map" [12]

$$
z=f_{1}(\zeta)=\mathrm{i} B\left[\frac{\partial}{\partial \alpha}-\frac{\partial}{\partial \bar{\alpha}}\right] \tilde{G}_{0}(\zeta ; \alpha)+C
$$

where $\tilde{G}_{0}(\zeta ; \alpha)$ is the analytic extension of the modified Green's function given in (23) and $B$ and $C$ are real constants. (This provides an interesting link between the covering map and the potential theory associated with the spectrum.) This alternative form is also discussed in [12]. The representation

$$
z(\zeta)=f_{2}(\zeta)=E\left[\frac{\omega^{2}(\zeta, 1)-\omega^{2}(\zeta,-1)}{\omega^{2}(\zeta, 1)+\omega^{2}(\zeta,-1)}\right]+F
$$

where $E$ and $F$ are real constants, is also viable. Formula (31) has been used in an application studying fluid motion through gaps in walls [16]. 


\section{Minimal Herglotz Functions}

From its characterization given in [9, Thm. 6.1], we know that a minimal Herglotz function $m(z)$

(a) is a rational function on the hyperelliptic curve $\mathcal{S}$;

(b) it has a simple pole at $\infty^{-}$and one more in each of the $l$ gaps;

(c) it also has a simple zero at $\infty^{+}$with asymptotic behaviour

$$
m(z)=-\frac{1}{z}-\frac{b_{1}}{z^{2}}-\frac{a_{1}^{2}+b_{1}^{2}}{z^{3}}+\mathcal{O}\left(1 / z^{4}\right)
$$

where $a_{1}$ and $b_{1}$ are Jacobi parameters. It also has one more zero in each of the $l$ gaps.

The procedure of "coefficient stripping" [9] corresponds to the following: with the function $m_{1}(z)$, say, yielding parameters $a_{1}$ and $b_{1}$ successfully found, the parameters $a_{2}$ and $b_{2}$ can be determined by constructing another minimal Herglotz function $m_{2}(z)$, say, with all the same properties of $m_{1}(z)$ but where the simple zeros of $m_{1}(z)$ in the gaps become the simple poles of next-generation Herglotz function $m_{2}(z)$. By iterating this construction, the full set of Jacobi parameters can be generated.

To effect this construction within our model, we introduce

$$
M(\zeta) \equiv m(z(\zeta))
$$

If $m(z)$ is rational on $\mathcal{S}$ then the function $M(\zeta)$ must be automorphic with respect to the Schottky group $\Theta$. It follows from the general theory for automorphic functions of this kind $[3,13]$ that we can write

$$
M(\zeta)=D\left[\frac{\omega(\zeta, \alpha)}{\omega(\zeta, 1 / \alpha)}\right] \prod_{k=1}^{l}\left[\frac{\omega\left(\zeta, s_{k}\right)}{\omega\left(\zeta, r_{k}\right)}\right] \mathrm{e}^{2 \pi \mathrm{i} \sum_{k=1}^{l} n_{k} v_{k}(\zeta)}
$$

for some set of integers $\left\{n_{k} \mid k=1, \ldots, l\right\}$ and some constant $D$ where $\omega(.,$.$) is the$ relevant Schottky-Klein prime function with the poles $\left\{1 / \alpha, r_{j} \mid j=1, \ldots, l\right\}$ and zeros $\left\{\alpha, s_{j} \mid j=1, \ldots, l\right\}$ chosen to satisfy a set of automorphicity conditions [13]-these are just the conditions of Abel's theorem [3]. It is clear from (34) that $M(\zeta)$ has the required simple pole at $\zeta=1 / \alpha$, corresponding to $z=\infty^{-}$, and that it vanishes at $\zeta=\alpha$, i.e. at $z=\infty^{+}$. Each point $\zeta=r_{j}$ is on the real axis in $F$ and projects down to the gap $\left[\beta_{j-1}, \alpha_{j}\right]$ for $j=1, \ldots, l$. On use of (14), these $l$ conditions can be expressed as

$$
v_{j}(\alpha)-v_{j}(1 / \alpha)+\sum_{k=1}^{l}\left(v_{j}\left(s_{k}\right)-v_{j}\left(r_{k}\right)\right)=-\sum_{k=1}^{l} n_{k} \tau_{k j}, \quad j=1, \ldots, l .
$$


Observe that the first factor in the representation (34) for the minimal Herglotz function can be written, on use of (24), in the form

$$
M(\zeta)=\tilde{D} \mathrm{e}^{2 \pi \mathrm{i} \tilde{G}_{0}(\zeta, \alpha)} \prod_{j=1}^{l}\left[\frac{\omega\left(\zeta, s_{j}\right)}{\omega\left(\zeta, r_{j}\right)}\right] \mathrm{e}^{2 \pi \mathrm{i} \sum_{k=1}^{l} n_{k} v_{k}(\zeta)}
$$

where $\tilde{D}$ is some constant and $\tilde{G}_{0}(\zeta, \alpha)$ is the modified Green's function of (23). A similar expression involving the potential theoretic Green's function is found using the alternative approach of [9]. Note that finding the zero set $\left\{s_{j}\right\}$ for a given set of poles from the expressions in (35) is an example of a Jacobi inversion problem. We proceed to present our construction under the assumption that its solution exists and is unique.

Finally, we also make a comment here on the integrals of the first kind for the classes of domains to which we have now restricted. For such domains we can always pick the real part of $v_{j}(\zeta)$, for each $j=1, \ldots, l$, for $\zeta$ on the real axis to be zero, so that

$$
\overline{v_{j}}(\zeta)=-v_{j}(\zeta)
$$

Together, (17) and (37) imply that

$$
v_{j}(1 / \zeta)=-v_{j}(\zeta), \quad j=1, \ldots, l
$$

\section{Inverse Problem for the Jacobi Parameters}

We can write

$$
M(\zeta)=\frac{N(\zeta)}{f(\zeta)}=\frac{N(\zeta)}{z}
$$

where

$$
N(\zeta) \equiv A D\left[\frac{\omega(\zeta, \beta) \omega(\zeta, 1 / \beta)}{\omega(\zeta, 1 / \alpha)^{2}}\right] \prod_{j=1}^{l}\left[\frac{\omega\left(\zeta, s_{j}\right)}{\omega\left(\zeta, r_{j}\right)}\right] \mathrm{e}^{2 \pi \mathrm{i} \sum_{k=1}^{l} n_{k} v_{k}(\zeta)}
$$

Since both $M(\zeta)$ and $f(\zeta)$ are automorphic with respect to the Schottky group action then so is $N(\zeta)$. A Taylor expansion of $N(\zeta)$ near $\zeta=\alpha$ gives

$$
N(\zeta)=N(\alpha)+(\zeta-\alpha) N^{\prime}(\alpha)+\frac{N^{\prime \prime}(\alpha)}{2 !}(\zeta-\alpha)^{2}+\cdots
$$

or, on use of (29),

$$
N=N(\alpha)+\frac{N^{\prime}(\alpha) X_{\infty}}{z}+\left[N^{\prime}(\alpha) X_{\infty} Y_{\infty}+\frac{X_{\infty}^{2} N^{\prime \prime}(\alpha)}{2 !}\right] \frac{1}{z^{2}}+\cdots
$$


On substitution of this into (39), we find

$$
M=\frac{N(\alpha)}{z}+\frac{N^{\prime}(\alpha) X_{\infty}}{z^{2}}+\left[N^{\prime}(\alpha) X_{\infty} Y_{\infty}+\frac{X_{\infty}^{2} N^{\prime \prime}(\alpha)}{2}\right] \frac{1}{z^{3}}+\cdots
$$

Enforcing the condition that $M=-1 / z+\mathcal{O}\left(1 / z^{2}\right)$ implies

$$
N(\alpha)=-1
$$

On substitution of this result into (43), we arrive at

$$
M=-\frac{1}{z}-\frac{X_{\infty} N^{\prime}(\alpha)}{N(\alpha)} \frac{1}{z^{2}}-\left[X_{\infty} Y_{\infty} \frac{N^{\prime}(\alpha)}{N(\alpha)}+\frac{X_{\infty}^{2}}{2} \frac{N^{\prime \prime}(\alpha)}{N(\alpha)}\right] \frac{1}{z^{3}}+\cdots
$$

A comparison with (32) yields

$$
b_{1}=\frac{X_{\infty} N^{\prime}(\alpha)}{N(\alpha)}, \quad a_{1}^{2}+b_{1}^{2}=X_{\infty} Y_{\infty} \frac{N^{\prime}(\alpha)}{N(\alpha)}+\frac{X_{\infty}^{2}}{2} \frac{N^{\prime \prime}(\alpha)}{N(\alpha)} .
$$

The logarithmic derivatives arising naturally in these expressions imply that they can be rewritten more explicitly as Eq. (4) which are independent of the prefactor $A D$ in the formula (40) for $N(\zeta)$ which is why we have set $A D=1$ in (5).

\section{Periodic Jacobi Matrices}

To examine the nature of any conditions necessary for the determination of a periodic Jacobi matrix, we now study a specific example. Modifications for the general case will be obvious from what follows.

Suppose that we seek a $p=4$ periodic Jacobi matrix in the $l=2$ gap case. Suppose that the initial poles are at $\left\{r_{1}, x_{1}\right\}$ with zeros, following from Abel's theorem, at $\left\{s_{1}, y_{1}\right\}$ (in addition to the pole at $1 / \alpha$ and zero at $\alpha$ that are present at every stage). Then, from (35), we have

$$
\begin{aligned}
& v_{1}(\alpha)-v_{1}(1 / \alpha)+v_{1}\left(s_{1}\right)-v_{1}\left(r_{1}\right)+v_{1}\left(y_{1}\right)-v_{1}\left(x_{1}\right)=-n_{11}^{(1)} \tau_{11}-n_{21}^{(1)} \tau_{21}, \\
& v_{2}(\alpha)-v_{2}(1 / \alpha)+v_{2}\left(s_{1}\right)-v_{2}\left(r_{1}\right)+v_{2}\left(y_{1}\right)-v_{2}\left(x_{1}\right)=-n_{12}^{(1)} \tau_{12}-n_{22}^{(1)} \tau_{22},
\end{aligned}
$$

for some integers $\left\{n_{j k}^{(1)}\right\}$. The construction via the minimal Herglotz functions implies that there are a succession of poles and zeros given by

$$
\begin{array}{ll}
\left\{1 / \alpha, s_{1}, y_{1}\right\} \text { (poles), } & \left\{\alpha, s_{2}, y_{2}\right\} \text { (zeros), } \\
\left\{1 / \alpha, s_{2}, y_{2}\right\} \text { (poles), } & \left\{\alpha, s_{3}, y_{3}\right\} \text { (zeros), } \\
\left\{1 / \alpha, s_{3}, y_{3}\right\} \text { (poles), } & \left\{\alpha, s_{1}, y_{1}\right\} \text { (zeros), }
\end{array}
$$


where since the zeros of the last iteration (aside from the one at $\alpha$ ) will now become the poles of the next minimal Herglotz function (aside from the one at $1 / \alpha$ ), and since the matrix is periodic by assumption, the sequence must repeat itself. The poles and zeros (48) satisfy, respectively, the Abel conditions

$$
\begin{aligned}
& v_{1}(\alpha)-v_{1}(1 / \alpha)+v_{1}\left(s_{2}\right)-v_{1}\left(s_{1}\right)+v_{1}\left(y_{2}\right)-v_{1}\left(y_{1}\right)=-n_{11}^{(2)} \tau_{11}-n_{21}^{(2)} \tau_{21}, \\
& v_{2}(\alpha)-v_{2}(1 / \alpha)+v_{2}\left(s_{2}\right)-v_{2}\left(s_{1}\right)+v_{2}\left(y_{2}\right)-v_{2}\left(y_{1}\right)=-n_{12}^{(2)} \tau_{12}-n_{22}^{(2)} \tau_{22},
\end{aligned}
$$

and

$$
\begin{aligned}
& v_{1}(\alpha)-v_{1}(1 / \alpha)+v_{1}\left(s_{3}\right)-v_{1}\left(s_{2}\right)+v_{1}\left(y_{3}\right)-v_{1}\left(y_{2}\right)=-n_{11}^{(3)} \tau_{11}-n_{21}^{(3)} \tau_{21}, \\
& v_{2}(\alpha)-v_{2}(1 / \alpha)+v_{2}\left(s_{3}\right)-v_{2}\left(s_{2}\right)+v_{2}\left(y_{3}\right)-v_{2}\left(y_{2}\right)=-n_{12}^{(3)} \tau_{12}-n_{22}^{(3)} \tau_{22},
\end{aligned}
$$

and

$$
\begin{aligned}
& v_{1}(\alpha)-v_{1}(1 / \alpha)+v_{1}\left(r_{1}\right)-v_{1}\left(s_{3}\right)+v_{1}\left(x_{1}\right)-v_{1}\left(y_{3}\right)=-n_{11}^{(4)} \tau_{11}-n_{21}^{(4)} \tau_{21}, \\
& v_{2}(\alpha)-v_{2}(1 / \alpha)+v_{2}\left(r_{1}\right)-v_{2}\left(s_{3}\right)+v_{2}\left(x_{1}\right)-v_{2}\left(y_{3}\right)=-n_{12}^{(4)} \tau_{12}-n_{22}^{(4)} \tau_{22}
\end{aligned}
$$

for some $\left\{n_{j k}^{(m)} \in \mathbb{Z} \mid j, k=1,2 ; m=1,2,3,4\right\}$. Addition of (47) and (49)-(51) produces a cancellation of most of the terms leaving, for $j=1,2$,

$$
p\left[v_{j}(\alpha)-v_{j}(1 / \alpha)\right]=\sum_{k=1}^{l} N_{k}^{(j)} \tau_{k j},
$$

where

$$
N_{k}^{(j)}=\sum_{m=1}^{p} n_{k j}^{(m)}
$$

is some integer. But (38) allows us to deduce that (52) is

$$
2 p v_{j}(\alpha)=\sum_{k=1}^{l} N_{k}^{(j)} \tau_{k j}, \quad \text { for } j=1, \ldots, l
$$

Hence, on use of (21), (54) can be written after division by $2 p$ as

$$
v_{j}(\alpha)=-i \sum_{k=1}^{l} \frac{N_{k}^{(j)}}{p} Q_{k j}, \quad \text { for } j=1, \ldots, l \text {. }
$$


On evaluating (20) at $\zeta=\alpha$, we find

$$
v_{j}(\alpha)=\sum_{k=1}^{l} Q_{j k} \tilde{v}_{k}(\alpha), \quad \text { for } j=1, \ldots, l .
$$

But the matrix $Q_{j k}$ is symmetric and invertible [15,28], so a comparison of (55) and (56) leads to

$$
\tilde{v}_{k}(\alpha) \equiv H_{k}(\alpha)+\mathrm{i} \Omega_{k}(\alpha)=-\frac{\mathrm{i} N_{k}^{(j)}}{p}, \quad \text { for } j=1, \ldots, l,
$$

which, on equating imaginary parts, implies that we must have

$$
\Omega_{k}(\alpha)=\frac{m_{k}}{p}
$$

for some integer $m_{k}$. Thus, the condition for a $p$-periodic Jacobi matrix is that the $l$ harmonic measures, when evaluated at infinity (i.e. at $\zeta=\alpha$ ), must be rational numbers of the form (58).

Such matters are explored in more detail in [9] within the covering map formalism considered there; other related discussion is to be found in [22, Cor. 5]. The above treatment aims to give a flavour of how these considerations translate to the Schottky uniformization used here.

\section{Two Spectral Bands}

An instructive exercise is to study the single gap case (genus one) within this Schottky model because the construction can be performed analytically, and quite explicitly. It is then possible to see both the construction based on our formulation in action, as well as many features of the underlying general theory [9]. All the required function theory can be derived from elementary considerations without invoking any special results from classical elliptic or theta function theory. The genus-one case has also been considered, from different perspectives, by other authors [1,26].

For the concentric annulus $\rho<|\zeta|<1$, the Schottky-Klein prime function is

$$
\omega(\zeta, \alpha)=-\frac{\alpha}{C} P(\zeta / \alpha)
$$

where $P(\zeta)$ can be defined by the infinite product

$$
P(\zeta) \equiv(1-\zeta) \prod_{n=1}^{\infty}\left(1-\rho^{2 n} \zeta\right)\left(1-\rho^{2 n} / \zeta\right), \quad C \equiv \prod_{n=1}^{\infty}\left(1-\rho^{2 n}\right)^{2}
$$

This is precisely the form given by (16) in this doubly connected case where the Schottky group has just a single generator (and its inverse). As for the prime function 
itself, we suppress the dependence of $P(\zeta)$ on the conformal modulus $\rho$. The product (60) can be shown to converge by standard methods and it is elementary to verify, directly from the infinite product definition, that $P(\zeta)$ satisfies

$$
P(1 / \zeta)=-\zeta^{-1} P(\zeta), \quad P\left(\rho^{2} \zeta\right)=-\zeta^{-1} P(\zeta)
$$

Indeed (60) is proportional to the infinite product given in (16) when the Schottky group $\Theta$ is generated by $\theta_{1}(\zeta)=\rho^{2} \zeta$ and its inverse. The second of the relations (61) is essentially just (14) with the function $v_{1}(\zeta)$ defined by

$$
v_{1}(\zeta)=\frac{1}{2 \pi i} \log \zeta, \quad \tau_{11}=\frac{1}{2 \pi i} \log \rho^{2}
$$

It is clear from (62), and its definition given earlier, that the single harmonic measure in this case is

$$
\Omega_{1}(\zeta)=\frac{\log |\zeta|}{\log \rho}
$$

In passing, we note that the Schottky-Klein prime function (59) can be related to the first Jacobi theta function (see [10,11]).

The conformal mapping from the concentric annulus $\rho<|\zeta|<1$ to two bands on the real axis is

$$
z(\zeta)=R\left[\frac{P(\zeta / \beta) P(\zeta \beta)}{P(\zeta / \alpha) P(\zeta \alpha)}\right]
$$

for some real $\alpha$ with $\rho<\alpha<1$ and real parameters $R, \rho$ and $\beta$. The choice of these four real parameters corresponds to the freedom to pick the four real end points of the two spectral bands.

Period-2 Solution Let us take the first minimal Herglotz function to be

$$
M_{1}(\zeta)=\frac{A_{1}}{\zeta}\left[\frac{P(\zeta / \alpha) P\left(\zeta / s_{1}\right)}{P(\zeta \alpha) P\left(\zeta / r_{1}\right)}\right]
$$

where the simple pole $r_{1}$ satisfies $-1 / \rho<r_{1}<-\rho$ so that it sits in the gap. The condition for automorphicity, i.e. the requirement that $M_{1}(\zeta)$ satisfies

$$
M_{1}\left(\rho^{2} \zeta\right)=M_{1}(\zeta)
$$

is easily seen to be

$$
\frac{\alpha^{2} s_{1}}{\rho^{2} r_{1}}=1, \quad \text { or } \quad \frac{s_{1}}{r_{1}}=\frac{\rho^{2}}{\alpha^{2}} .
$$

For a period-2 solution the second minimal Herglotz function is then

$$
M_{2}(\zeta)=A_{2}\left[\frac{P(\zeta / \alpha) P\left(\zeta / r_{1}\right)}{P(\zeta \alpha) P\left(\zeta / s_{1}\right)}\right]
$$


where, in order that this is automorphic, we require

$$
\frac{r_{1} \alpha^{2}}{s_{1}}=1, \quad \text { or } \quad \frac{s_{1}}{r_{1}}=\alpha^{2} .
$$

Together (67) and (69) imply the condition

$$
\alpha^{2}=\rho
$$

which is consistent with the requirement that $\rho<\alpha<1$.

On taking a logarithm, condition (70) is

$$
\frac{\log |\alpha|}{\log \rho}=\frac{1}{2}
$$

which, by (63), is equivalent to

$$
\Omega_{1}(\alpha)=\frac{1}{2}
$$

Period-3 Solution Let the first minimal Herglotz function be

$$
M_{1}(\zeta)=\frac{A_{1}}{\zeta}\left[\frac{P(\zeta / \alpha) P\left(\zeta / s_{1}\right)}{P(\zeta \alpha) P\left(\zeta / r_{1}\right)}\right]
$$

with $-1 / \rho<r_{1}<-\rho$. Again we must enforce the condition (67) for automorphicity. Now suppose the second minimal Herglotz function is

$$
M_{2}(\zeta)=A_{2}\left[\frac{P(\zeta / \alpha) P\left(\zeta / s_{2}\right)}{P(\zeta \alpha) P\left(\zeta / s_{1}\right)}\right] .
$$

Then, that it is automorphic, we require

$$
\frac{s_{2} \alpha^{2}}{s_{1}}=1, \quad \text { or } \quad \frac{s_{2}}{s_{1}}=\frac{1}{\alpha^{2}} .
$$

Finally, for a period-3 solution, the third minimal Herglotz function is

$$
M_{3}(\zeta)=A_{3}\left[\frac{P(\zeta / \alpha) P\left(\zeta / r_{1}\right)}{P(\zeta \alpha) P\left(\zeta / s_{2}\right)}\right],
$$

where, for its automorphicity, we require

$$
\frac{r_{1} \alpha^{2}}{s_{2}}=1, \quad \text { or } \quad \frac{r_{1}}{s_{2}}=\frac{1}{\alpha^{2}} .
$$

It follows from (67), (75) and (77) that we must have

$$
\alpha^{3}=\rho
$$


which is consistent with the requirement that $\rho<\alpha<1$. On taking logarithms this is equivalent, by (63), to

$$
\Omega_{1}(\alpha)=\frac{1}{3}
$$

But there is another option. Instead of choosing $M_{2}(\zeta)$ as in (80) we could alternatively pick

$$
M_{2}(\zeta)=\frac{A_{2}}{\zeta}\left[\frac{P(\zeta / \alpha) P\left(\zeta / s_{2}\right)}{P(\zeta \alpha) P\left(\zeta / s_{1}\right)}\right],
$$

implying the automorphicity condition

$$
\frac{\alpha^{2} s_{2}}{\rho^{2} s_{1}}=1, \quad \text { or } \quad \frac{s_{2}}{s_{1}}=\frac{\rho^{2}}{\alpha^{2}} .
$$

Then, with $M_{3}(\zeta)$ given by (76), conditions (67), (81) and (77) imply

$$
\alpha^{3}=\rho^{2},
$$

which is consistent with the requirement that $\rho<\alpha<1$. On taking logarithms this is equivalent, by (63), to

$$
\Omega_{1}(\alpha)=\frac{2}{3}
$$

\section{Two Spectral Bands of Equal Length}

The case of two spectral bands of equal length is of interest and has recently been studied, in a more general context, by Eichingeret al. [21]. Our formulation can be applied to this special case. The condition (70) for a period-two Jacobi matrix is consistent with a spectrum comprising two equal intervals. To see this, consider the covering map given by

$$
z=f(\zeta)=R \frac{P(-\zeta / \sqrt{\rho}) P(-\zeta \sqrt{\rho})}{P(\zeta / \sqrt{\rho}) P(\zeta \sqrt{\rho})} .
$$

It is readily verified that this map satisfies

$$
f(\rho / \zeta)=-f(\zeta)
$$

implying the required symmetry between the images of $|\zeta|=\rho$ and $|\zeta|=1$.

For two spectral bands on the real axis between $\left[-(\lambda+2)^{1 / 2},-(\lambda-2)^{1 / 2}\right]$ and $\left[(\lambda-2)^{1 / 2},(\lambda+2)^{1 / 2}\right]$ where $\lambda>2$ we must pick $\rho$ such that

$$
\frac{f(+1)}{f(-1)}=\left(\frac{\lambda+2}{\lambda-2}\right)^{1 / 2}
$$


and, for given $\lambda$, this is easily solved by Newton's method. Once $\rho$ is determined $R$ follows explicitly:

$$
R=(\lambda+2)^{1 / 2}\left[\frac{P(1 / \sqrt{\rho}) P(\sqrt{\rho})}{P(-1 / \sqrt{\rho}) P(-\sqrt{\rho})}\right] .
$$

With the covering map determined by the geometry of the spectral bands in this way, it can be shown that

$$
X_{\infty}=-\frac{R \sqrt{\rho} P(-1) P(-\rho)}{\hat{P}(1) P(\rho)}, \quad Y_{\infty}=\frac{X_{\infty}}{2 \sqrt{\rho}}
$$

where we define

$$
\hat{P}(\zeta) \equiv \prod_{n=1}^{\infty}\left(1-\rho^{2 n} \zeta\right)\left(1-\rho^{2 n} / \zeta\right)
$$

In addition, with

$$
\begin{aligned}
& N_{1}(\zeta) \equiv M_{1}(\zeta) f(\zeta)=\frac{P(-\zeta / \sqrt{\rho}) P(-\zeta \sqrt{\rho}) P\left(\zeta /\left(\rho r_{1}\right)\right)}{\zeta P^{2}(\zeta \sqrt{\rho}) P\left(\zeta / r_{1}\right)}, \\
& N_{2}(\zeta) \equiv M_{2}(\zeta) f(\zeta)=\frac{P(-\zeta / \sqrt{\rho}) P(-\zeta \sqrt{\rho}) P\left(\zeta / r_{1}\right)}{P^{2}(\zeta \sqrt{\rho}) P\left(\zeta /\left(\rho r_{1}\right)\right)},
\end{aligned}
$$

where we have used the expressions for $M_{1}(\zeta)$ and $M_{2}(\zeta)$ from (65) and (68), it follows directly that

$$
\begin{aligned}
\left.\frac{\mathrm{d}}{\mathrm{d} \zeta} \log N_{1}\right|_{\zeta=\sqrt{\rho}}=\frac{1}{\sqrt{\rho}} & {\left[K(-1)+K(-\rho)+K\left(1 /\left(\sqrt{\rho} r_{1}\right)\right)\right.} \\
& \left.-1-2 K(\rho)-K\left(\sqrt{\rho} / r_{1}\right)\right] \\
\left.\frac{\mathrm{d}}{\mathrm{d} \zeta} \log N_{2}\right|_{\zeta=\sqrt{\rho}}=\frac{1}{\sqrt{\rho}} & {\left[K(-1)+K(-\rho)+K\left(\sqrt{\rho} / r_{1}\right)\right) } \\
& \left.-2 K(\rho)-K\left(1 /\left(\sqrt{\rho} r_{1}\right)\right)\right]
\end{aligned}
$$

where

$$
K(\zeta) \equiv \frac{\zeta P^{\prime}(\zeta)}{P(\zeta)}
$$

which, from (61), can be shown to satisfy

$$
K\left(\rho^{2} \zeta\right)=K(\zeta)-1, \quad K(1 / \zeta)=1-K(\zeta)
$$

On use of the results (easily checked) that $K(-1)=1 / 2, K( \pm \rho)=0$, we find

$$
b_{1}=-b_{2} \text {. }
$$


With a second differentiation, we find

$$
\begin{aligned}
& \left.\frac{\mathrm{d}^{2}}{\mathrm{~d} \zeta^{2}} \log N_{1}\right|_{\zeta=\sqrt{\rho}}=-\frac{1}{\rho}\left[K(-1)+K(-\rho)+K\left(1 /\left(\sqrt{\rho} r_{1}\right)\right)\right. \\
& \left.-1-2 K(\rho)-K\left(\sqrt{\rho} / r_{1}\right)\right] \\
& +\frac{1}{\rho}\left[L(-1)+L(-\rho)+L\left(1 /\left(\sqrt{\rho} r_{1}\right)\right)\right. \\
& \left.-2 L(\rho)-L\left(\sqrt{\rho} / r_{1}\right)\right], \\
& \left.\frac{\mathrm{d}^{2}}{\mathrm{~d} \zeta^{2}} \log N_{2}\right|_{\zeta=\sqrt{\rho}}=-\frac{1}{\rho}\left[K(-1)+K(-\rho)+K\left(\sqrt{\rho} / r_{1}\right)\right) \\
& \left.-2 K(\rho)-K\left(1 /\left(\sqrt{\rho} r_{1}\right)\right)\right] \\
& +\frac{1}{\rho}\left[L(-1)+L(-\rho)+L\left(\sqrt{\rho} / r_{1}\right)\right) \\
& \left.-2 L(\rho)-L\left(1 /\left(\sqrt{\rho} r_{1}\right)\right)\right]
\end{aligned}
$$

where we define

$$
L(\zeta) \equiv \zeta K^{\prime}(\zeta)
$$

It can be confirmed numerically that, for any choice of $r_{1}$,

$$
a_{1}=\frac{1}{a_{2}} .
$$

In summary, we find the following structure of period-2 Jacobi matrices over two equal intervals:

$$
\left[\begin{array}{cccccccc}
\cdot & \cdot & \cdot & & & & & \\
a & b & \frac{1}{a} & & & & \\
0 & \frac{1}{a} & -b & a & & & \\
& & a & b & \frac{1}{a} & & \\
& & & \frac{1}{a} & -b & a & \\
& & & & & & & \\
& & & & & \cdot & \cdot
\end{array}\right]
$$

where $a$ and $b$ are real parameters given by

$$
\begin{aligned}
b & =\frac{X_{\infty}}{\sqrt{\rho}}\left[K(-1)+K(-\rho)+K\left(1 /\left(\sqrt{\rho} r_{1}\right)\right)-1-2 K(\rho)-K\left(\sqrt{\rho} / r_{1}\right)\right], \\
a^{2} & =Y_{\infty} b-\frac{b^{2}}{2}
\end{aligned}
$$




$$
\begin{gathered}
+\frac{X_{\infty}^{2}}{2}\left\{-\frac{1}{\rho}\left[K(-1)+K(-\rho)+K\left(1 /\left(\sqrt{\rho} r_{1}\right)\right)-1-2 K(\rho)-K\left(\sqrt{\rho} / r_{1}\right)\right]\right. \\
\left.+\frac{1}{\rho}\left[L(-1)+L(-\rho)+L\left(1 /\left(\sqrt{\rho} r_{1}\right)\right)-2 L(\rho)-L\left(\sqrt{\rho} / r_{1}\right)\right]\right\} .
\end{gathered}
$$

For a given spectrum, the parameters $\rho, X_{\infty}$ and $Y_{\infty}$ will be set leaving the parameters $a$ and $b$ in (99) dependent on the explicit way given above on the single real parameter $r_{1}$. Indeed, we are also able to confirm numerically that, for any $r_{1}$, the formulas for $a$ and $b$ given in (99) satisfy the relation

$$
\left(a-\frac{1}{a}\right)^{2}+b^{2}-(\lambda-2)=0
$$

established in an appendix to [21].

Just as $P(\zeta)$ can be related to the first Jacobi theta function, the functions $K(\zeta)$ and $L(\zeta)$ relate to the Weierstrass $\zeta$ and $\wp$ functions, respectively, so our results (99) can be viewed as expressions of the Jacobi parameters in terms of those special functions.

\section{Discussion}

This paper has presented the basis of a constructive approach to the inverse problem for finite gap Jacobi matrices based on a Schottky model of the underlying hyperelliptic spectral curve and use of the Schottky-Klein prime function. The latter function can now be calculated with great speed and computational efficiency by means of novel numerical algorithms (see $[15,17,20])$ ) which are not based on the calculation of infinite products over the Schottky group action, or of any infinite Blaschke products. While, for the groups relevant here, all those products are known to converge (for example, by some of Beardon's results - see [9] for references), they are not numerically efficient as a basis for constructive methods. The single gap case can be performed quite explicitly with our approach using a convenient infinite product formula for the Schottky-Klein prime function associated with a concentric annulus. The construction for more spectral bands can be performed entirely analogously, and with great numerical efficiency given the new software routines to evaluate Schottky-Klein prime functions on the Schottky doubles of planar domains [20].

A closing remark is that our explicit form (4) for the Jacobi parameters solving the inverse problem for a given spectrum is interesting. The appearance of a second derivative of $\log N(\zeta)$ is highly reminiscent of similar formulas in the isospectral theory of the $\mathrm{KdV}$ equation with the automorphic function $N(\zeta)$ ostensibly playing the role of a tau function.

Acknowledgements The author acknowledges support from an Established Career Fellowship from the Engineering and Physical Sciences Research Council (EPSRC) and from a Wolfson Research Merit Award from the Royal Society. He is also grateful for the hospitality of the Centre for Quantum Geometry of 
Moduli Spaces at the University of Aarhus, Denmark. This work was inspired by a masterclass delivered there by Prof. Barry Simon in February 2014 and attended by the author.

\section{Compliance with ethical standards}

Funding statement The research in this article is supported by an Established Career Fellowship from the Engineering and Physical Sciences Research Council (EP/K019430/1).

Open Access This article is distributed under the terms of the Creative Commons Attribution 4.0 International License (http://creativecommons.org/licenses/by/4.0/), which permits unrestricted use, distribution, and reproduction in any medium, provided you give appropriate credit to the original author(s) and the source, provide a link to the Creative Commons license, and indicate if changes were made.

\section{References}

1. Akhieser, N.I.: Über einige Funktionen, die in gegebenen Intervallen am wenigsten von Null abweichen. Bull. Soc. Phys. Math. Kazan. Ser. 3 3(2), 1-69 (1928)

2. Aptekarev, A.I.: Asymptotic properties of polynomials orthogonal on a system of contours, and periodic motions of Toda lattices. Math. USSR Sb. 53(1), 233-260 (1984)

3. Baker, H.F.: Abelian Functions: Abel's Theorem and the Allied Theory of Theta Functions. Cambridge University Press, Cambridge (1897)

4. Beardon, A.F.: A primer on Riemann surfaces. In: London Mathematical Society Lecture Note Series, vol. 78. Cambridge University Press, Cambridge (1984)

5. Bogatyrëv, A.B.: Prime form and Schottky model. Comput. Methods Funct. Theory 9(1), 47-55 (2009)

6. Bogatyrëv, A.B.: Extremal Polynomials and Riemann Surfaces. Springer, Berlin (2012)

7. Burnside, W.: On functions determined from their discontinuities and a certain form of boundary condition. Proc. Lond. Math. Soc. 22, 346-358 (1891)

8. Burnside, W.: On a class of automorphic functions. Proc. Lond. Math. Soc. 23, 49-88 (1892)

9. Christiansen, J.S., Simon, B., Zinchenko, M.: Finite gap Jacobi matrices, I: the isospectral torus. Const. Approx. 32, 1-65 (2010)

10. Crowdy, D.G.: Geometric function theory: a modern view of a classical subject. Nonlinearity 21(10), T205-T219 (2008)

11. Crowdy, D.G.: Conformal slit maps in applied mathematics. ANZIAM J. 53(3), 171-189 (2012)

12. Crowdy, D.G., Marshall, J.S.: Conformal mappings between canonical multiply connected domains. Comput. Methods Funct. Theory 6(1), 59-76 (2006)

13. Crowdy, D.G., Marshall, J.S.: On the construction of multiply connected quadrature domains. SIAM J. Appl. Math. 64, 1334-1359 (2004)

14. Crowdy, D.G., Marshall, J.S.: Analytical formulae for the Kirchhoff Routh path function in multiply connected domains. Proc. R. Soc. A 461, 2477-2501 (2005)

15. Crowdy, D.G., Marshall, J.S.: Computing the Schottky-Klein prime function on the Schottky double of planar domains. Comput. Methods Funct. Theory 7(1), 293-308 (2007)

16. Crowdy, D.G., Marshall, J.S.: The motion of a point vortex through gaps in walls. J. Fluid Mech. 551, 31-48 (2006)

17. Crowdy, D.G.: http://www2.imperial.ac.uk/ dgcrowdy/SKPrime

18. Crowdy, D.G., Marshall, J.S.: Green's functions for Laplace's equation in multiply connected domains. IMA J. Appl. Math. 72, 278-301 (2007)

19. Crowdy, D.G.: Vortex patch equilibria of the Euler equation and random normal matrices. J. Phys. A Math. Theor. 47, 212002 (2014)

20. Crowdy, D.G., Green, C.C., Kropf, E., Nasser, M.M.S.: The Schottky-Klein prime function: a theoretical and computational tool for applications. IMA J. Appl. Math. 81(3), 589-628 (2016)

21. Eichinger, B., Puchhammer, F., Yuditskii, P.: Jacobi flow on SMP matrices and Killip-Simon problem on two disjoint intervals. Comput. Methods Funct. Theory 16, 3-41 (2016). doi:10.1007/ s40315-014-0104-9

22. Lukashov, A., Peherstorfer, F.: Automorphic orthogonal and extremal polynomials. Can. J. Math. 55(2), 576-608 (2003) 
23. Goluzin, G.M.: Geometric Theory of Functions of a Complex Variable. American Mathematical Society, Providence (1969)

24. Gustafsson, B.: Quadrature identities and the Schottky double. Acta Appl. Math. 1(3), 209-240 (1983)

25. Hejhal, D.A.: Theta functions, kernel functions and Abelian integrals. Mem. Am. Math. Soc. 129. American Mathematical Society, Providence (1972)

26. Pavlov, B.S., Fedorov, S.I.: The group of shifts and harmonic analysis on a Riemann surface of genus one. Leningr. Math. J. 1(2), 447-490 (1990)

27. Peherstorfer, F., Yuditskii, P.: Asymptotic behaviour of polynomials orthonormal on a homogeneous set. J. Anal. Math. 89, 113-154 (2003)

28. Schiffer, M.: Recent advances in the theory of conformal mapping, appendix to: R. Dirichlet's Principle, Conformal Mapping and Minimal Surfaces. Interscience Publishers, New York (1950)

29. Sodin, M., Yuditskii, P.: Almost periodic Jacobi matrices with homogeneous spectrum, infinitedimensional Jacobi inversion, and Hardy spaces of character-automorphic functions. J. Geom. Anal. 7, 387-435 (1997)

30. Varchenko, A.N., Etingof, P.I.: University Lecture Series. Why the boundary of a round drop becomes a curve of order four. American Mathematical Society, Providence (1992)

31. Vasconcelos, G., Marshall, J.S., Crowdy, D.G.: Secondary Schottky-Klein prime functions associated with multiply connected planar domains. Proc. R. Soc. A 471, 2014688 (2014) 